\title{
BEAM OPTICS ISSUES FOR THE ANTIPROTON DECELERATOR
}

\author{
P. Belochitskii, JINR, RU-141 980 Dubna, Russia \\ C. Carli, T. Eriksson, R. Giannini, S. Maury, D. Möhl, F. Pedersen, \\ CERN, CH-1211 Geneva 23, Switzerland
}

\section{Abstract}

The deceleration of the beam down to $0.1 \mathrm{GeV} / \mathrm{c}$ in the ring previously used as Antiproton Collector (AC) at 3.5 $\mathrm{GeV} / \mathrm{c}$, requires a number of modifications to the lattice. The insertion of the electron cooling, needed to cool the antiproton beam at low energy, implies the re-arrangement of quadrupoles. The optical functions then need to be readjusted in order to keep the large acceptance and to cope with the electron and stochastic cooling environment. Calculations of the linear optics and of the acceptance are reported. Tests of beam deceleration in the AC show the need for closed-orbit correction at low momentum in addition to the static correction by the movement of the quadrupoles available in the present configuration. The deceleration tests will be discussed and a correction system, which includes trim supplies on the main bending magnets, will be described.

\section{THE ELECTRON COOLING INSERTION}

The present AC lattice [1] is made of 28 FODO cells with two straight sections of about $28 \mathrm{~m}$ length each, two of $15 \mathrm{~m}$ length and four densely packed arcs. The $28 \mathrm{~m}$ straight sections have no orbit dispersion whereas the $15 \mathrm{~m}$ sections have a small dispersion. To satisfy the topology imposed by the injection and ejection lines special 'half-quadrupoles' are used in the injection/ extraction section and some quadrupoles are transversally displaced in the other $28 \mathrm{~m}$ straight section in order to maintain symmetry.

For efficient operation as an Antiproton Decelerator [2,3] electron cooling is needed at low energy. The electron cooling device should be located in a straight section where the dispersion is zero and beta functions of
5-10 $\mathrm{m}$ are desired. To gain space for the cooler, the central quadrupole of the long straight section opposite to the injection section is removed and the two adjacent Fquadrupoles are shifted towards the next D-lenses. The rematching of the optics is done by decreasing the distance between the closest two lenses on either side of the cooler.

The required strength for these new D-lenses is beyond the values obtainable with the AC quadrupoles, so two identical quadrupoles are needed side by side using AC spares. The new layout of this section in shown in Fig. 1.

\section{AD LATTICE}

The very large acceptance requirements are $\Delta p / p= \pm 3 \%$ and $A_{h}=A_{v}=240 \pi \mathrm{mm} \cdot \mathrm{mrad}$, as in the present AC, in order to capture about $5 \times 10^{7} \overline{\mathrm{p}} /$ shot. Other conditions such as the phase advance between the pick-ups and the kickers of the stochastic cooling systems, and between injection/ejection septum and kickers, have also to be maintained close to their present values.

The optical functions are shown in Fig. 2. The horizontal envelope is larger $\left(\beta_{H} \cong 18 \mathrm{~m}\right.$ instead of $12 \mathrm{~m}$ in the AC) and the vertical envelope is similar to the one of the AC except in the first quadrupole of the cooling insertion where $\beta_{V}$ is $20 \mathrm{~m}$. For both machines, the dispersion function remains the same.

The working point $\left(Q_{h}=5.28, Q_{v}=5.19\right.$ instead of $Q_{h} \cong Q_{v} \cong 5.45$ in the $\mathrm{AC}$ ) has been chosen as a result of an optimization of the acceptance in the presence of the cooling insertion and the other constraints mentioned.

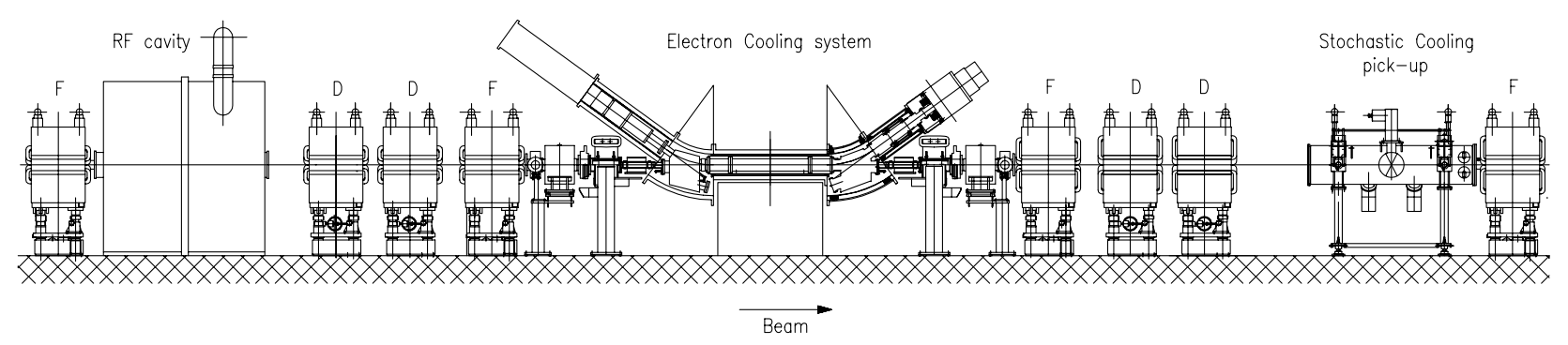

Fig.1: Layout of the Electron Cooling insertion. 


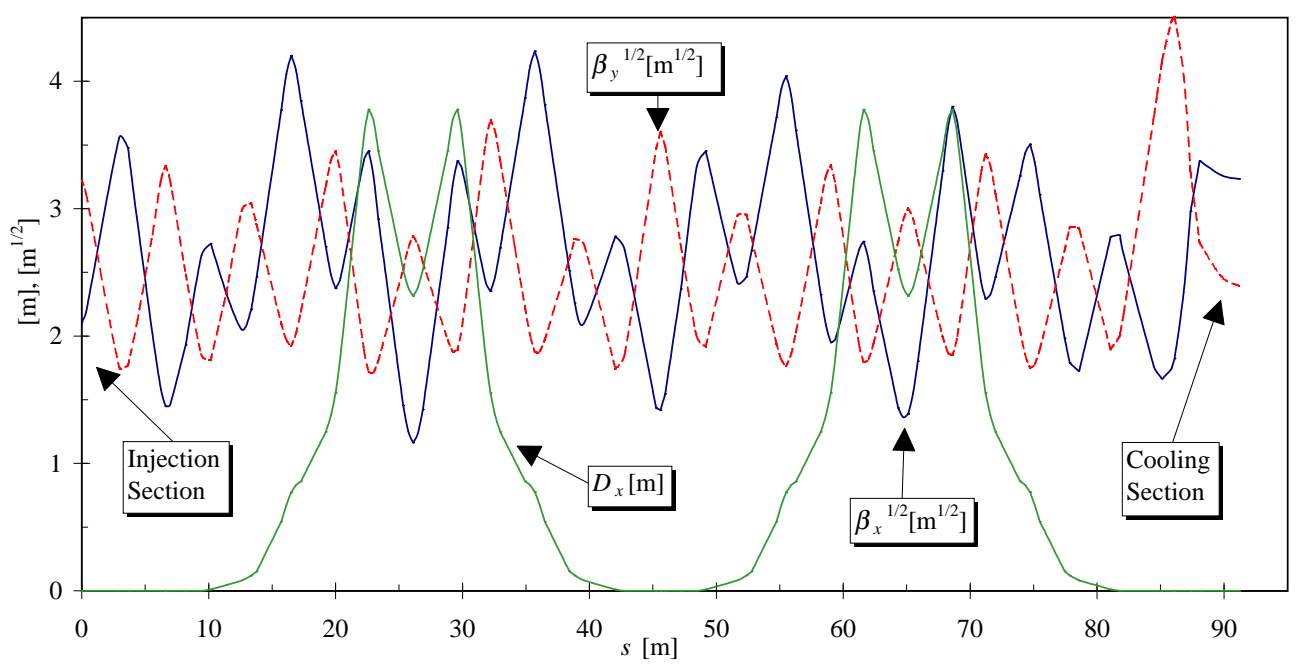

Fig. 2: Envelope functions $\left(\sqrt{\beta_{h}}, \sqrt{\beta_{v}}\right)$ and dispersion function $D$ for the AD. The plot is for one half of the ring, starting in the middle of the injection section and finishing in the cooling section.

\section{TESTS ON THE AC}

Machine study sessions in parallel with the physics runs during 1995/1996 were used to decelerate a test beam of about $10^{9}$ protons to the lowest possible momentum and to identify the problems. A few decelerations were done with antiprotons $\left(N=5 \times 10^{7}\right)$ to demonstrate that it is feasible, but most decelerations were done with protons $\left(N=5 \times 10^{9}\right)$, where beam diagnostics to measure tunes and orbits are available. For that, the existing tunable ferrite cavity $(1.6 \mathrm{MHz}$, normally used for rebunching of antiprotons prior to extraction from the AC) was modified to cover a frequency range of 1 to $2 \mathrm{MHz}$ and a field sensing coil was installed in one of the ring bending magnets. A software package was written to control the ramping magnet currents which are adjusted based on beam measurements.

A plot of the fraction of the beam surviving after deceleration to different momenta is shown in Fig. 3. The lowest beam momentum obtained with enough protons left to measure orbits and tunes was $12 \%$ of maximum momentum $(430 \mathrm{MeV} / \mathrm{c})$. The ramping from $100 \%$ to $20 \%$ in this experiment took approx. 120 s. The design goal for the $\mathrm{AD}$ machine is deceleration to $3 \%$ in about $10 \mathrm{~s}$. We believe that the remaining losses are a combination of transverse emittance blow-up due to poor vacuum and reduced acceptance due to poor orbit. The limiting factors at $430 \mathrm{MeV} / \mathrm{c}$ were:

- Quad-trim power supply regulation working below a current of about $2 \mathrm{~A}$.

- Slow deceleration (software ramping) combined with poor vacuum leading to excessive emittance blow-up due to multiple Coulomb scattering.

- Tune measurements impossible with present system (50 MHz Schottky pick-up).
- Poor tracking (current regulation) limiting deceleration speed.

- Stability of field sensing coil and/or B-train generator.

- Reduced acceptance due to orbit errors.

Both horizontal and vertical orbits were measured as a function of momentum.

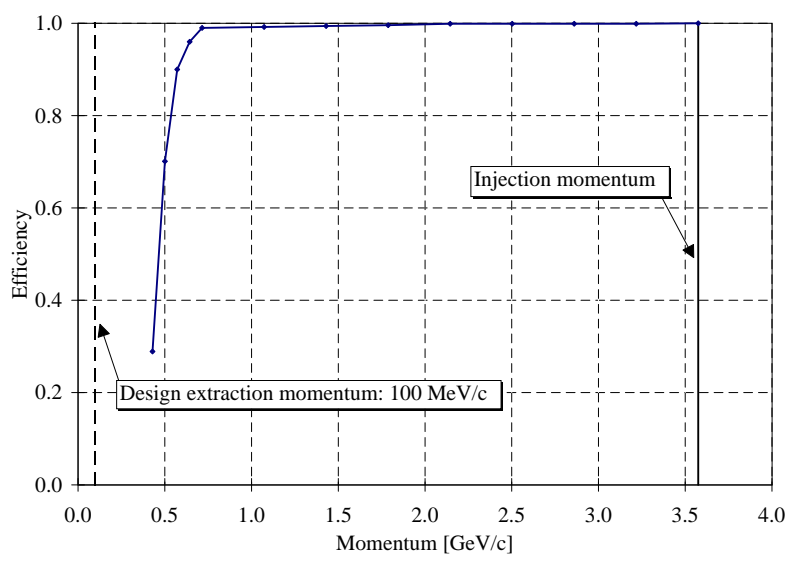

Fig. 3: Beam survival during deceleration of a pencil beam of about $10^{9}$ protons as a function of momentum.

These experiments clearly showed the need for closed-orbit correction at low energy. In fact, horizontal excursions of $\pm 30 \mathrm{~mm}$ (Fig. 4) were measured at 430 $\mathrm{MeV} / \mathrm{c}$ even though the orbit was carefully corrected at $3.57 \mathrm{GeV} / \mathrm{c}$ by the present 'static' system of moving the quadrupoles. The orbit variations are probably due to saturation asymmetries at high energy and to remanence asymmetries at low energies.

The measured beam positions in the straight sections down to $430 \mathrm{MeV} / \mathrm{c}$ are shown in Fig. 4. 


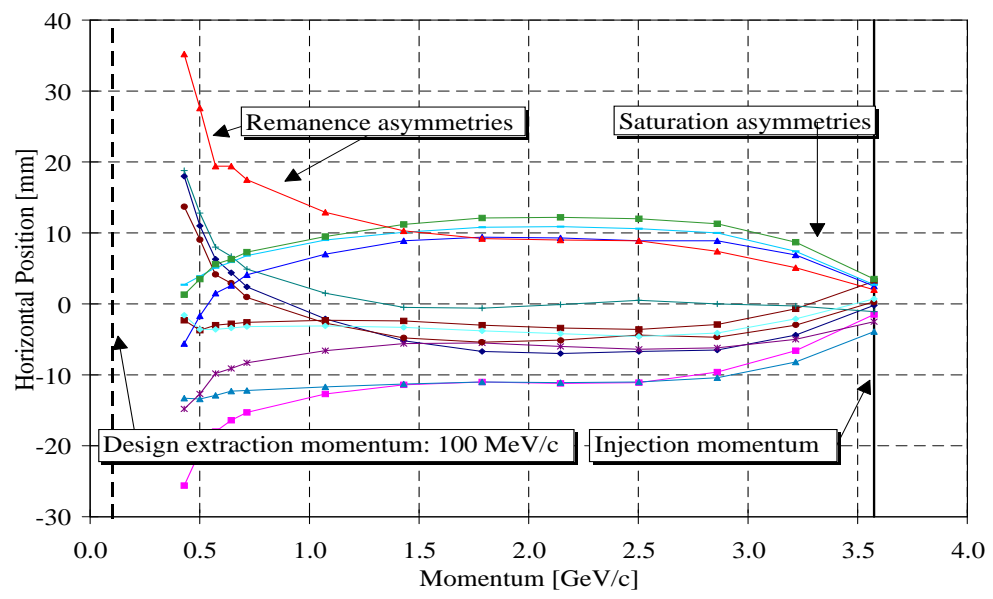

Fig. 4: Horizontal orbit distortion vs. momentum measured at 12 pick-ups in the AC. At $3.57 \mathrm{GeV} / \mathrm{c}$ the orbit was corrected (acceptance optimized) by adjustment of the quadrupole positions.

\section{ORBIT CORRECTION}

To extrapolate the orbit distortions to low energies a formula: $\chi_{0}=A+B / p$ was fitted through all, but the two highest measured momenta. Extrapolation to $100 \mathrm{MeV} / \mathrm{c}$ indicated distortions up to $\pm 150 \mathrm{~mm}$ and $\pm 15 \mathrm{~mm}$ for the horizontal and vertical planes, respectively.

The scheme retained for horizontal orbit correction is based on trim supplies that will be available on the bending magnets plus a few additional correctors that will be installed in the long straight section and especially on both sides of the electron cooling insertion.

It has been decided to connect the 24 magnets in groups of adjacent pairs thus needing a total of 12 trimming supplies. Application of a MICADO algorithm [4] gives the residual distortion of the horizontal orbit at $100 \mathrm{MeV} / \mathrm{c}$ smaller than $15 \mathrm{~mm}$, as indicated in Fig. 5.

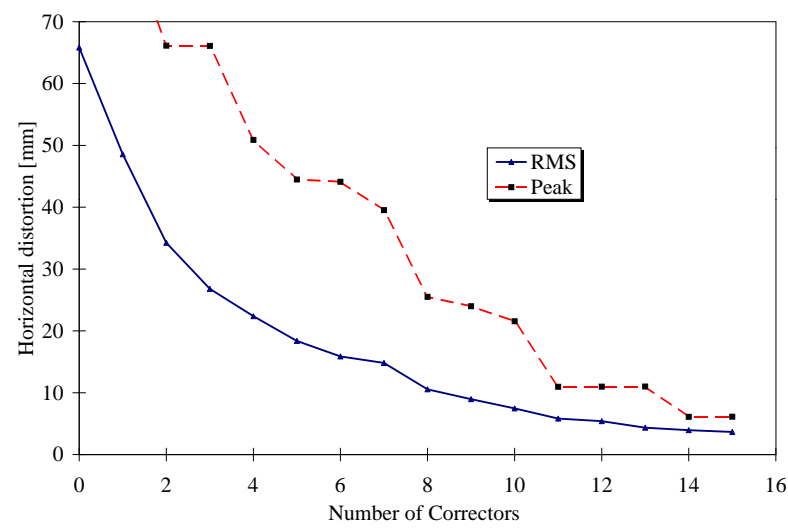

Fig. 5: Residual distortion of the horizontal orbit (in $\mathrm{mm}$ ) at the pick-up locations after correction using $n$ correctors in the AC.

With 14 vertical correction dipoles, installed in locations where space can be made available, the vertical closed-orbit distortions at $100 \mathrm{MeV} / \mathrm{c}$ are reduced from $15 \mathrm{~mm}$ to about $4 \mathrm{~mm}$.

Together with the static correction (quadrupole movement), this system can be used for an orbit correction to better than $\pm 15 \mathrm{~mm}$ horizontally and $\pm 5 \mathrm{~mm}$ vertically in the entire energy range, at least for the errors extrapolated from the present AC.

\section{CONCLUSION}

All the identified limitations will be eliminated by appropriate upgrades during the course of the AD project. Modification of the AC lattice and an orbit correction scheme have been found which should permit electron cooling and efficient beam deceleration to $100 \mathrm{MeV} / \mathrm{c}$. Work is underway to confirm and improve the scheme.

\section{REFERENCES}

[1] H. Koziol and S. Maury, 'Parameter List for the Antiproton Accumulator Complex (AAC)', Edition 1994, CERN/PS 95-15, 1995.

[2] S. Baird, D. Berlin, J. Boillot, J. Bosser, M. Brouet, J. Buttkus, F. Caspers, V. Chohan, D. Dekkers, T. Eriksson, R. Garoby, R. Giannini, O. Grobner, J. Gruber, J.Y. Hémery, H. Koziol, R. Maccaferri, S. Maury, C. Metzger, K. Metzmacher, D. Möhl, H. Mulder, M. Paoluzzi, F. Pedersen, J.P. Riunaud, C. Serre, D.J. Simon, G. Tranquille, J. Tuyn, B. Williams: 'Design Study of the Antiproton Decelerator: AD', CERN/PS/96-43 (AR), 1996.

[3] S. Baird, D. Berlin, J. Boillot, J. Bosser, M. Brouet, J. Buttkus, F. Caspers, V. Chohan, D. Dekkers, T. Eriksson, R. Garoby, R. Giannini, O. Grobner, J. Gruber, J.Y. Hémery, H. Koziol, R. Maccaferri, S. Maury, C. Metzger, K. Metzmacher, D. Möhl, H. Mulder, M. Paoluzzi, F. Pedersen, J.P. Riunaud, C. Serre, D.J. Simon, G. Tranquille, J. Tuyn, and A. Van der Schueren: 'The Antiproton Decelerator (AD)', this conference.

[4] B. Autin, Y. Marti, 'Closed Orbit Correction of A.G. Machines Using a Small Number of Magnets', CERN-ISR-MA/73-17, 1973. 